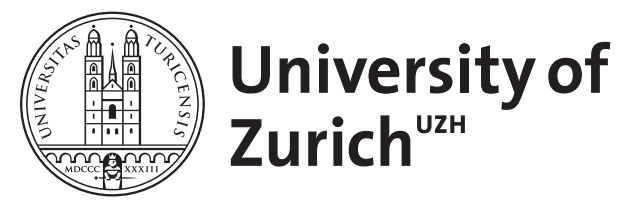

\title{
Progress in neuro-otology research in the last year
}

\author{
Tarnutzer, A A ; Straumann, D
}

\begin{abstract}
Herein, we summarize articles in the field of neuro-otology published in the Journal of Neurology over the last year. Topics included acute and chronic vertigo as well as auditory and ocular motor disorders. Characteristic lesion locations in Pusher syndrome are reported and the usefulness of bedside ocular motor tests in vertebrobasilar stroke is revisited. Probing the vestibular system and its value in predicting the outcome in vegetative state is discussed. Several articles address new diagnostic and therapeutic approaches in different disorders associated with chronic vestibular, auditory or gait deficits. In a series of case reports, we focus on different eye movement disorders in the vertical plane, which are often difficult to assess.
\end{abstract}

DOI: https://doi.org/10.1007/s00415-012-6670-8

Posted at the Zurich Open Repository and Archive, University of Zurich

ZORA URL: https://doi.org/10.5167/uzh-65402

Journal Article

Accepted Version

Originally published at:

Tarnutzer, A A; Straumann, D (2012). Progress in neuro-otology research in the last year. Journal of Neurology, 259(11):2506-2509.

DOI: https://doi.org/10.1007/s00415-012-6670-8 
MEDICAL PROGRESS IN THE JOURNAL OF NEUROLOGY

Progress in neuro-otology research in the last year

\author{
Alexander A. Tarnutzer, Dominik Straumann \\ Department of Neurology, University Hospital Zurich, Switzerland
}

Corresponding author:
A.A. Tarnutzer
Department of Neurology
University Hospital Zurich
Frauenklinikstrasse 26
CH-8091 Zurich
Switzerland

Alexander.Tarnutzer@access.uzh.ch 


\begin{abstract}
Herein, we summarize articles in the field of neuro-otology published in the Journal of Neurology over the last year. Topics included acute and chronic vertigo as well as auditory and ocular motor disorders. Characteristic lesion locations in Pusher syndrome are reported and the usefulness of bedside ocular motor tests in vertebrobasilar stroke is revisited. Probing the vestibular system and its value in predicting the outcome in vegetative state is discussed. Several articles address new diagnostic and therapeutic approaches in different disorders associated with chronic vestibular, auditory or gait deficits. In a series of case reports, we focus on different eye movement disorders in the vertical plane, which are often difficult to assess.
\end{abstract}




\section{Keywords}

neuro-otology, eye movements, nystagmus, ocular motor disorders 


\section{Introduction}

Herein, main articles describing novel findings in the field of neuro-otology published in the Journal of Neurology over the last year are summarized. This review includes articles on ocular motor disorders due to lesions of central vestibular pathways and structures. 


\section{Acute vertigo}

The differential diagnosis for both short-lasting episodic and prolonged acute vertigo / dizziness is broad. Expert opinion suggests focusing on detailed history taking and a thorough neurological examination with a focus on distinguishing potentially dangerous and benign self-limiting conditions. The acute vestibular syndrome or AVS (defined as acute prolonged vertigo / dizziness for more than 24 hours accompanied by nausea / vomiting, nystagmus, impaired balance) demands a differentiation between benign peripheral (e.g. vestibular neuritis) and more dangerous central (e.g. PICA-stroke or cerebellar hemorrhage) causes. While the horizontal head-impulse test has $100 \%$ sensitivity for detecting central AVS, it is only about $90 \%$ specific, as $10 \%$ of the ponto-cerebellar and inferior cerebellar strokes will present with a positive head-impulse test due to either labyrinthitis or root entry zone ischemia. In 2009, Kattah and colleagues suggested that a combination of three bedside ocular motor tests (horizontal head-impulse testing, evaluation for skew deviation and directionchanging gaze-evoked horizontal nystagmus) is superior to MRI including DWI obtained within the first 48 to 72 hours. Three articles published in the Journal of Neurology in the past year address different aspects of central AVS. Chen et al. (1) added a fourth bedside test (evaluation of vertical smooth pursuit) and prospectively examined the diagnostic accuracy of these four bedside tests and neuroimaging over the period of one year in a stroke unit. Finding one of the bedside signs of a central origin of AVS was 100\% sensitive and $90 \%$ specific for stroke, which is comparable to those previously reported by expert neuro-otologists. The practical implication from this study could be that unnecessary imaging can be spared in patients with peripheral-type AVS based on bedside examination.

In a single case report with a very unusual stroke topography, Scocco et al. (2) showed how near simultaneous altered gravitational perception, unilateral proprioceptive loss, and impaired postural compensation may delay recovery from central AVS. Poor compensation was associated with the multi-modal impairment of both afferent and efferent tracts by combined posterior fossa (left-cerebellar and medullary) and spinal (left posterior column) stroke, affecting graviceptive-vestibular and somatosensory inputs and compensatory extensor muscle tone adaptation by vestibulo-spinal and reticulo-spinal tracts.

Ischemic strokes leading to acute postural imbalance are addressed in an article by Baier et al. (3), focusing on 27 patients with active pushing away from the non-paralyzed side (also 
termed Pusher Syndrome). Based on their findings, the authors suggested that insular, opercular as well as temporal regions are possibly involved in the control of body upright position. Furthermore they postulated a link (with stronger experimental support for rightsided lesions) between the neuronal networks in charge of postural control and those for the processing of vestibular otolith input. With regards to the multisensory integration at specialized cortical areas, such close interaction between postural control and graviceptive input makes perfect sense. 


\section{Chronic vertigo}

While patients with a first episode of acute vertigo may functionally recover, a significant number of patients present with chronic dizziness / vertigo often of unknown origin. Such chronic vertigo may be associated with a previous episode of acute vertigo or not, however, this distinction is sometimes difficult to achieve. Based on two single cases with acute vestibular neuritis without peripheral recovery, Brandt et al. (4) discuss the clinical relevance of artificial neural network posturography in differentiating between a secondary transition to phobic postural vertigo and complaints related to incomplete functional recovery of peripheral vestibular function. While their first case - having developed new symptoms of subjective postural and gait unsteadiness after 5 months - was rated with a high probability as suffering from phobic postural vertigo by the artificial neural network, their second case reflected complaints consistent with incomplete recovery of peripheral vestibular function. Thus the authors conclude that artificial neural network posturography may differentiate with a high sensitivity and specificity between residually impaired peripheral vestibular function and secondary phobic postural vertigo and therefore helps in identifying those patients who would benefit from behavioral therapy.

In nearly half of the patients with dizziness/vertigo that persists for more than 6 months investigated at specialized dizzy clinics the complaints are not fully explained by an identifiable medical illness and are related to phobic, panic, anxiety, depressive, dissociative, or somatoform disorders. Lack of understanding of the underlying pathophysiological mechanisms and lack of predictors for developing dizziness / vertigo related to a psychiatric disorder led to the initiation of the Munich Diagnostic and Predictor Study of Dizziness, which was designed to investigate diagnostic subgroups, correlates, and predictors of dizziness that is not sufficiently explained medically, but clearly related to a psychiatric disorder (5). This study is ongoing and the patient assessment will consist of a baseline and two follow-up assessment points at 6 and 12 months in a total of approximately 800 patients based on power analysis. Eventually, the data gathered are expected to improve the diagnosis and treatment of patients with dizziness related to a psychiatric disorder.

Recurrent disequilibrium / vertigo, auditory (diplacusis, autophony) and visual symptoms (oscillopsia due to torsional nystagmus) often triggered by loud noises of low frequency or middle-ear pressure changes, has been referred to as the Tullio phenomenon. Many of these 
cases are associated with a superior semicircular canal dehiscence (SSCD), leading to inappropriate stimulation of the affected semicircular canal by generating a "third" mobile window in the labyrinth with a low resistance pathway for transmission of low frequency sound energy through the labyrinth. In some Tullio patients, however, high-resolution CT imaging of the temporal bones does not confirm a SSCD, pointing to other possible underlying causes of Tullio's phenomenon such as a perilymphatic fistula or enlarged vestibular aqueduct. Treatment options include avoidance of precipitants, surgical occlusion or resurfacing of the affected canal, all of which can ameliorate symptoms. Kaski et al. (6) review the current literature and report three cases with a new clinical syndrome of unknown etiology consisting of Tullio's phenomenon with bilateral vestibular failure, normal cervical vestibular-evoked myogenic potentials (cVEMPs), a pure horizontal sound-induced nystagmus and no evidence for canal dehiscence.

Persistent habituation to passive background movements may result in the rare, but pathophysiologically very interesting condition of mal de debarquement syndrome (MdDS), which is related to the erroneous percept of self-motion, most frequently observed following a cruise. MdDS therefore appears to be a disorder of neuroplasticity and sensory rearrangement, which explains why the quantification of cortical excitability by use of single-pulse and paired-pulse transcranial magnetic stimulation as reported by Clark and Quick (7) in a single patient with persistent MdDS is of interest. The elevated levels of intracortical facilitation along with the low levels of short-interval intracortical inhibition and the shorter silent period compared to age-matched controls led the authors to the hypothesis that MdDS may result in alterations in the balance of intracortical properties towards hyperexcitability, which would be in accordance with the reported benefit from substances lowering intracortical facilitation such as benzodiazepines. Alternatively - as pointed out by the authors - hyperexcitability may predispose to the development of MdDS rather than reflecting neuroplasticity induced by MdDS.

Chronic impairment of balance leading to ataxia of gait and stance is a frequent clinical sign in a variety of progressive disorders including spino-cerebellar ataxias, Friedreich's ataxia, episodic ataxia and others. This combination of clinical signs may also be cause by Hashimoto's encephalitis - a condition otherwise closely linked to altered cognition. With gait ataxia, weakness, dysmetria and dysarthria being the prominent feature in a patient with steroid-responsive encephalopathy associated with autoimmune thyroiditis (or Hashimoto 
encephalitis) and lack of signs of encephalopathy, Tang et al. (8) conclude that the spectrum of clinical presentation of Hashimoto encephalitis can be broader than previously thought, including signs of spino-cerebellar ataxia. 


\section{Auditory disorders}

Chronic tinnitus still presents a difficult to treat condition in many patients. Current concepts of the pathophysiology of tinnitus suggest both abnormal activity in central auditory pathways and deficient inhibitory control from the prefrontal cortex, with the latter potentially modulating its attentional and emotional aspects. In an open-label pilot study Frank et al. (9) addressed the question whether repeated application of bifrontal transcranial direct current stimulation (tDCS) would result in a longer-lasting reduction of tinnitus. The therapeutic effects of bifrontal tDCS on chronic tinnitus observed were only minor and were genderdependent, demonstrating better effects for female patients. With regards to the main outcome parameter - differences in the tinnitus handicap inventory score before and after treatment repeated bifrontal tDCS did not show any benefit.

Whether auditory dysfunction is part of the spectrum of neurological abnormalities associated with Leber's hereditary optic neuropathy (LHON) and what perceptual consequences would arise from an auditory neuropathy in affected patients is not known. Rance et al. (10) provided evidence for functional hearing difficulties due to a neural abnormality in the central auditory pathways in $>25 \%$ of patients with the LHON genetic profile (either symptomatic or asymptomatic with regards to optic neuropathy), advocating auditory testing including auditory evoked potentials and speech perception in all LHON patients. At the same time, cochlear function was normal or near-normal. 


\section{Ocular motor disorders}

Because of the vestibulo-ocular reflex (VOR), which elicits short-latency compensatory eye movements in response to vestibular stimulation, lesions of peripheral or central vestibular pathways and structures frequently lead to abnormal eye movements. In the acute state, ocular motor disorders are often associated with symptoms of vertigo, while abnormal eye movements maybe the chief neuro-otologic findings in the chronic state.

Patients with cerebellar degeneration frequently show downbeat nystagmus (DBN). It is well known that slow-phase eye velocity of DBN modulates with the position of the head relative to gravity in the pitch plane. Typically, DBN increases when patients bend forward. Sander et al. (11) demonstrated that the beneficial effect of the potassium-channel blocker 4aminopyridine (4-AP) is most striking when DBN is measured with the head bent forward. 4AP may partly restore cerebellar inhibition of the overacting otolith-ocular reflex in patients with DBN.

Besides DBN, other types of vertical nystagmus point to specific lesions within the brainstem or cerebellum. Carota et al. (12) described a patient who developed vertical pendular nystagmus (documented with video) three months after a pontine hemorrhage. T2-weighted MRI showed the typical finding of hypertrophic inferior olivary nuclei degeneration (HIOND). Baclofen, pregabalin, memantine, and clonazepam did not dampen the pendular nystagmus; with lamotrigine it became even worse. Progress in the treatment of ocular HIOND manifestations is still awaited. A comprehensive review on the pharmacotherapy of vestibular and ocular motor disorders, including pendular nystagmus, was recently published by Strupp et al. (13).

Bassani et al. (14) reported a patient suffering from pendular nystagmus with phase-shifted vertical and horizontal components. This nystagmus was associated with progressive cerebellar ataxia and palatal tremor (all signs documented with video). T2-weighted MRI showed increased signal intensity in the superior cerebellar peduncles and HIOND. This case demonstrates that pendular nystagmus in HIOND is not always purely vertical.

Neugebauer et al. (15) presented a patient, in whom upbeat nystagmus (documented with video) and impaired consciousness was noted after she was given physostigmine. Upbeat 
nystagmus was synchronously linked to right-sided occipital epileptic spikes. Epileptic nystagmus therefore can also be directed vertically and not only horizontally, as in most cases reported so far.

Kunte et al. (16) published the first report of a patient with Algrove syndrome who developed Wernicke's encephalopathy including upbeat nystagmus and horizontal gaze palsy. Algrove or AAA (alacrima, achalasia and adrenocorticotrophic hormone resistant adrenal insufficiency) syndrome is a rare autosomal-recessive disease. Wernicke's encephalopathy usually results from thiamine deficiency, which in this patient was probably due to achalasiainduced malnutrition. T2-weighted FLAIR MRI showed signal elevation of the periaqueductal gray matter and of the medial thalamus on both sides, while DWI MRI demonstrated hyperintense signals within both mamillary bodies.

Vertical gaze palsy usually point to midbrain lesions. Pothalil and Gille (17) reported the rare case of a patient with purely unilateral rostral midbrain infarction (as revealed on DWI-MRI), but conjugate vertical gaze palsy both upward and downward. The authors speculated that a unilateral midbrain lesions that include the rostral interstitial nucleus of the medial longitudinal fasciculus (riMLF) and the interstitial nucleus of Cajal (INC) as well as decussating fibers to and from these the same structures on the other side, is sufficient to explain the vertical gaze palsy.

Caloric irrigation is often used in intensive care units to assess brainstem function. Upon irrigation one can distinguish among three possibilities: no response, ocular drift (i.e. no fast phases), nystagmus (i.e. both slow and fast phases). Weiss et al. (18) applied cold-water caloric irrigation (10 deg Celsius, $60 \mathrm{ml}$ ) in patients who were in vegetative state (VS) due to brain injury. While all of the 13 patients who regained consciousness showed caloric nystagmus, this nystagmus was only seen in 1 of the 13 patients who remained unconscious. In 10 of these patients, caloric irrigation elicited ocular drift towards the side of the cold-water irrigation, but no fast phases. Therefore, the presence of caloric nystagmus in VS patients seems to be a good predictor for subsequently regaining consciousness. 


\section{References}

1. Chen L, Lee W, Chambers BR, Dewey HM. Diagnostic accuracy of acute vestibular syndrome at the bedside in a stroke unit. J Neurol 2011, May;258(5):855-61.

2. Scocco D, Pula JH, Kattah JC. Contralateral axial lateropulsion and ocular tilt reaction in a cerebello-lateral medullary-spinal stroke. J Neurol 2012, Mar;259(3):551-3.

3. Baier B, Janzen J, Müller-Forell W, Fechir M, Müller N, Dieterich M. Pusher syndrome: Its cortical correlate. J Neurol 2012, Feb;259(2):277-83.

4. Brandt T, Strupp M, Novozhilov S, Krafczyk S. Artificial neural network posturography detects the transition of vestibular neuritis to phobic postural vertigo. J Neurol 2012, Jan;259(1):182-4.

5. Lahmann C, Henningsen P, Dieterich M, Feuerecker R, Cyran CA, Schmid G. The munich diagnostic and predictor study of dizziness: Objectives, design, and methods. J Neurol 2012, Apr;259(4):702-11.

6. Kaski D, Davies R, Luxon L, Bronstein AM, Rudge P. The tullio phenomenon: A neurologically neglected presentation. J Neurol 2012, Jan;259(1):4-21.

7. Clark BC, Quick A. Exploring the pathophysiology of mal de debarquement. J Neurol 2011, Jun;258(6):1166-8.

8. Tang Y, Chu C, Lin MT, Wei G, Zhang X, Da Y, et al. Hashimoto's encephalopathy mimicking spinocerebellar ataxia. J Neurol 2011, Sep;258(9):1705-7.

9. Frank E, Schecklmann M, Landgrebe M, Burger J, Kreuzer P, Poeppl TB, et al. Treatment of chronic tinnitus with repeated sessions of prefrontal transcranial direct current stimulation: Outcomes from an open-label pilot study. J Neurol 2012, Feb;259(2):327-33.

10. Rance G, Kearns LS, Tan J, Gravina A, Rosenfeld L, Henley L, et al. Auditory function in individuals within leber's hereditary optic neuropathy pedigrees. J Neurol 2012, Mar;259(3):542-50.

11. Sander T, Sprenger A, Marti S, Naumann T, Straumann D, Helmchen C. Effect of 4aminopyridine on gravity dependence and neural integrator function in patients with idiopathic downbeat nystagmus. J Neurol 2011, Apr;258(4):618-22.

12. Carota A, Düron N, Cereda C, Bassetti CL. Vertical pendular nystagmus and hypertrophic inferior olivary nuclei degeneration: An "odd couple". J Neurol 2012, Feb;259(2):372-4.

13. Strupp M, Thurtell MJ, Shaikh AG, Brandt T, Zee DS, Leigh RJ. Pharmacotherapy of vestibular and ocular motor disorders, including nystagmus. J Neurol 2011, Jul;258(7):1207-22. 
14. Bassani R, Mariotti C, Nanetti L, Grisoli M, Savoiardo M, Pareyson D, Salsano E. Pendular nystagmus in progressive ataxia and palatal tremor. J Neurol 2011, Oct;258(10):1877-9.

15. Neugebauer H, Winkler T, Feddersen B, Pfister HW, Noachtar S, Straube A, Pfefferkorn T. Upbeat nystagmus as a clinical sign of physostigmine-induced right occipital non-convulsive status epilepticus. J Neurol 2012, Apr;259(4):773-4.

16. Kunte H, Nümann A, Ventz M, Siebert E, Harms L. Wernicke's encephalopathy in a patient with triple A (allgrove) syndrome. J Neurol 2011, Oct;258(10):1882-4.

17. Pothalil D, Gille M. Conjugate downward and upward vertical gaze palsy due to unilateral rostral midbrain infarction. J Neurol 2012, Apr;259(4):779-82.

18. Weiss N, Tadie JM, Faugeras F, Diehl JL, Fagon JY, Guerot E. Can fast-component of nystagmus on caloric vestibulo-ocular responses predict emergence from vegetative state in ICU? J Neurol 2012, Jan;259(1):70-6. 